\title{
Prevalência de intercorrências na gestação em mulheres acompanhadas na atenção primária à saúde
}

\author{
Prevalence of intercorrences in pregnancy in women accompanied in primary health care \\ Prevalencia de intercorrencias en el embarazo en mujeres seguidas en atención primaria \\ de salud
}

Andressa Prates Sá ${ }^{1 *}$, Amanda Lopes Franco ${ }^{1}$, Thais Pereira Silva ${ }^{1}$, Cristiane Aparecida Pires Fernandes ${ }^{1}$, Darilene Agda Lima dos Santos ${ }^{1}$, Dayane Araújo Rocha ${ }^{2}$, Keilla Silva Santos ${ }^{1}$, Karine Suene Mendes Almeida Ribeiro', Ingrid Isabel de Andrade', Sélen Jaqueline Souza Ruas'.

\section{RESUMO}

Objetivo: Identificar a prevalência das intercorrências obstétricas. Métodos: O estudo apresentou caráter retrospectivo, descritivo de corte transversal e análise quantitativa. Participaram da pesquisa as mulheres maiores de 18 anos, que tiveram gravidez e parto nos últimos 6 meses e que fossem cadastradas e acompanhadas pela Estratégia Saúde da Família de um município do Norte de Minas Gerais. Para coleta de dados foi utilizado o Google Formulário com a elaboração de um questionário desenvolvido pelas próprias pesquisadoras. Resultados: Em relação às principais intercorrências foi verificado que $27,3 \%$ das participantes tiveram infecção urinária durante a gravidez e 27,3\% tiveram diabetes gestacional. Sobre a doença hipertensiva da gestação $22,7 \%$ tiveram, e a toxoplasmose teve apenas $4,5 \%$. Em relação a doenças infecciosas, três participantes afirmaram que tiveram candidíase durante a gravidez (13,6\%). Nenhuma das participantes nunca teve Sífilis e placenta prévia. Dentre outras intercorrências prevaleceu o colo do útero curto com (4,5\%). Conclusão: Foi considerado um número significativo de mulheres que tiveram algum tipo de intercorrência durante a gravidez, sendo fator de risco para predispor a outras complicações. Nessa pesquisa constatou-se que as intercorrências que mais acometeram as participantes foram infecção urinária, diabetes mellitus gestacional e distúrbios hipertensivos.

Palavras-chave: Complicações na gravidez, Complicações do trabalho de parto, Cuidado pré natal.

\begin{abstract}
Objective: To identify the prevalence of obstetric complications. Methods: the study was retrospective, crosssectional descriptive and quantitative analysis. Women over 18 years old, who had pregnancy and childbirth in the last 6 months and who were registered and monitored by the Family Health Strategy of a municipality in the North of Minas Gerais, participated in the research. For data collection, Google Form was used with the elaboration of a questionnaire developed by the researchers themselves. Results: Regarding the main complications, it was found that $27.3 \%$ of the participants had urinary tract infections during pregnancy and $27.3 \%$ had gestational diabetes. About the hypertensive disease of pregnancy $22.7 \% \mathrm{had}$, and toxoplasmosis had only $4.5 \%$. Regarding infectious diseases, three participants stated that they had candidiasis during pregnancy $(13.6 \%)$. None of the participants never had syphilis and placenta previa. Among other complications, the short cervix prevailed (4.5\%). Conclusion: It was considered a significant number of women who had some type of complication during pregnancy, being a risk factor for predisposing to other complications. In this research, it was found that the complications that most affected the participants were urinary tract infections, gestational diabetes mellitus and hypertensive disorders.
\end{abstract}

Keywords: Pregnancy complications, Obstetric labor complications, Prenatal care.

\footnotetext{
1 Faculdade de Saúde e Humanidades Ibituruna (FASI), Montes Claros - MG.

*E-mail: andressaprates92@gmail.com

2 Universidade do Estado de Minas Gerais (UEMG), Passos - MG.
} 


\section{RESUMEN}

Objetivo: Identificar la prevalencia de complicaciones obstétricas. Métodos: El estudio fue retrospectivo, análisis descriptivo transversal y cuantitativo. Participaron de la investigación mujeres mayores de 18 años, que tuvieron embarazo y parto en los últimos 6 meses y que fueron registradas y monitoreadas por la Estrategia de Salud de la Familia de un municipio del Norte de Minas Gerais. Para la recogida de datos se utilizó Google Form con la elaboración de un cuestionario desarrollado por los propios investigadores. Resultados: En cuanto a las principales complicaciones, se encontró que el 27,3\% de las participantes presentaba infecciones del tracto urinario durante el embarazo y el 27,3\% tenía diabetes gestacional. Sobre la enfermedad hipertensiva del embarazo, el $22,7 \%$ la tenía y la toxoplasmosis sólo el 4,5\%. Respecto a las enfermedades infecciosas, tres participantes manifestaron haber tenido candidiasis durante el embarazo (13,6\%). Ninguno de los participantes nunca tuvo sífilis ni placenta previa. Entre otras complicaciones, predominó el cuello uterino corto $(4,5 \%)$. Conclusión: Se consideró un número significativo de mujeres que presentaron algún tipo de complicación durante el embarazo, siendo un factor de riesgo de predisposición a otras complicaciones. En esta investigación se encontró que las complicaciones que más afectaron a las participantes fueron las infecciones del tracto urinario, la diabetes mellitus gestacional y los trastornos hipertensivos.

Palabras clave: Complicaciones del embarazo, Complicaciones del trabajo de parto, Cuidado prenatal.

\section{INTRODUÇÃO}

A gravidez é uma fase que acarreta diversas mudanças na vida da mulher, no que se refere à adaptações fisiológicas, emocionais, psíquicas e sociais, as quais necessitam de atenção integral e assistência no binômio mãe-filho. Por mais que a gestante siga todas as recomendações que a ela foram passadas nas consultas, sendo ela de qualidade, ainda assim, haverá riscos de intercorrências (MINISTÉRIO DA SAÚDE, 2018a).

A assistência pré-natal tem por objetivo controlar a morbimortalidade materno-fetal. Exames clínicos e laboratoriais oferecidos durante as consultas de pré-natal possibilitam identificar situações de risco e atuar precocemente, mais adiante a assistência no momento do parto, indispensável determinante para morbimortalidade no decorrer do período neonatal (SILVA EP, et al., 2016).

Intercorrências são eventos inesperados durante a gestação ou no parto, podendo levar à morte materna e fetal devido a essas complicações. De acordo com a Organização Pan-Americanas da Saúde (OPAS) e Organização Mundial da Saúde (OMS) (2018), aproximadamente 830 mulheres no mundo evoluíram para óbito por fatores preveníveis devido ao ciclo gravídico-puerperal. Dentre as alterações mais frequentes, estão Infecções do Trato Urinário (ITU), a Doença Hipertensiva da Gestação (DHEG), anemia e hiperemese, doenças infectocontagiosas, cardiopatias ou endocrinopatias (diabetes mellitus gestacional, tireoideopatias), distúrbios do líquido amniótico, hemorragias, doenças infecciosas, Trabalho de Parto Prematuro (TPP), placenta prévia (VARELA PLR, et al., 2017).

Houve um número maior de internações por complicações obstétricas em mulheres no pós-parto, do que anterior ao parto, respectivamente, 3,8 vezes mais frequentes (MOURA BLA, et al., 2018). Devido a alterações fisiológicas no corpo da mulher, o sangramento no início da gravidez é considerado aceitável, acometendo $8,1 \%$ dentre as gestantes no primeiro trimestre da gravidez (CIRQUEIRA GC, et al., 2018). Porém, no decorrer da gestação é uma condição clínica grave e deve-se ser avaliada, pois quando se trata do acompanhamento de contrações uterinas, dilatação do colo e dores, a gravidez certamente será de alto risco e consequentemente ocorrerá um aborto espontâneo (MINISTÉRIO DA SAÚDE, 2012a).

A hemorragia pós-parto (HPP) é caracterizada como sangramento igual ou acima de $500 \mathrm{ml}$ (parto via baixa) e acima de $1000 \mathrm{ml}$ (parto via alta) (MINISTÉRIO DA SAÚDE, 2018b). Devido ser um grupo vulnerável, as multigestas têm maior chance de desenvolver esse quadro clínico (SHELDON WR, et al., 2014). Há possibilidades de causas da HPP, dentre elas estão a atonia uterina, lacerações de trajeto e a retenção de fragmentos placentários (MINISTÉRIO DA SAÚDE, 2001). 
A ITU na maioria das vezes pode ser assintomática e por isso muitas mulheres não procuram tratamento, se não tratada tem um potencial risco para pielonefrite e cistite, trazendo sérios prejuízos para o feto como seu nascimento antes de completar as 37 semanas, e seu período perinatal pequeno para idade gestacional (PIG) (MINISTÉRIO DA SAÚDE, 2012b).

A anemia na gestação pode implicar prejuízos como baixo peso, mortalidade perinatal, aumento de risco de aborto espontâneo, trabalho de parto premature (CAMPIGOTTO AC, et al., 2015). Pode-se atingir 19\% das grávidas no mundo (VARELA PLR, et al., 2017). O diabetes mellitus gestacional acomete 37,7\% dos casos no mundo, podendo levar a anomalias congênitas e consequentemente ao risco de aborto. Durante o desenvolvimento fetal pode ocorrer macrossomia, icterícia e evoluir à morte (BUCHANAN TA, et al., 2012). A DHEG pode causar danos irreversíveis, percentual de 5 a 17\% (OLIVEIRA ACM, et al., 2016). Se não tratada pode ocasionar a pré-eclâmpsia, com potencial risco de óbito fetal (SECRETARIA DE SAÚDE DO PARANÁ, 2018).

Em virtude dos benefícios que são ofertados, é essencial a gestante seguir todo o acompanhamento do pré-natal, visando a detecção precoce de doenças com possíveis prevenções, através da realização de exames para detecção de distúrbios metabólicos e hematológicos, urocultura, detecção de infecções sexualmente transmissíveis (MINISTÉRIO DA SAÚDE, 2016).

O pré-natal feito de maneira correta tem vantagens que favorecem a saúde da mulher não só no período gravídico, mas por vários anos. As consultas têm que seguir um cronograma, mediante a idade gestacional (IG): até 28 semanas, mensais; de 28 à 39 semanas, quinzenais; de 36 à 41 semanas e 6 dias, semanais. As consultas têm que se investigativas com intuito de correlatar agravos às condições estruturais de cada gestante de forma holística e deve ter a garantia de identificar disfunções patológicas que progridem de forma não explícita (NASCIMENTO DS, et al., 2021).

A consulta de pré-natal não deve focar apenas nos aspectos fisiológicos e no desenvolvimento do feto, outros fatores também são de extrema importância como oferecer informações necessárias à gestante quanto aos tipos de parto, as dores e ao estresse devido às alterações no qual o pico de hormônios causa no corpo e orientá-la sobre os sinais prodrômicos (MINISTÉRIO DA SAÚDE, 2016). É de suma importância a realização das consultas de pré-natal, intercorrências previsíveis não são vistas apenas pelo olhar da gestante e sim pelo conjunto de aspectos e classificações que se tem na consulta de pré-natal (MOURA BLA, et al., 2018).

A melhor forma de prevenir as intercorrências obstétricas é ter uma assistência de pré-natal o mais precoce possível, sob os cuidados e monitorização de profissionais qualificados, acolhendo a gestante com suas dúvidas, diminuindo assim, o seu medo, ansiedade e curiosidades. Deve-se orientá-la sobre a importância de seguir todos os cuidados que a ela serão passados, como, a importância da vacinação, não fazer uso de medicamentos sem prescrição médica, quanto a sexualidade sobre os riscos de Infecções Sexualmente Transmissíveis (IST's), álcool e drogas, realização de exames, sinais e sintomas do parto (MINISTÉRIO DA SAÚDE, 2016).

Devido a alterações fisiológicas no corpo da mulher, o sangramento no início da gravidez é considerado aceitável, acometendo 8,1\% dentre as gestantes no primeiro trimestre da gravidez (CIRQUEIRA GC, et al., 2018). Porém, no decorrer da gestação é uma condição clínica grave e deve-se ser avaliado, pois quando se trata do acompanhamento de contrações uterinas, dilatação do colo e dores, a gravidez certamente será de alto risco e consequentemente ocorrerá um aborto espontâneo (MINISTÉRIO DA SAÚDE, 2012).

O Pré-natal é ofertado pela Atenção Básica, que é considerada a principal porta de entrada para o SUS e refere-se como um conjunto de intervenções que inclui-se promoção, prevenção, proteção, diagnóstico, tratamento e reabilitação. Essas ações possuem intuito da continuidade de integralidade do cuidado. $A$ Atenção Básica tem uma habilidade de resolutividade dos problemas de saúde no primeiro nível de atenção e referência os usuários para outros níveis caso seja essencial (NASCIMENTO DS, et al.,2021).

É comprovado que quando a gestante tem uma assistência pré-natal qualificada reduz os riscos de intercorrências e a mortalidade materna e fetal. As compreensões desses aspectos podem melhorar a 
assistência de maneira geral, dando enfoque a saúde da gestante importante fator de saúde pública. Assim, este estudo teve como objetivo identificar a prevalência das intercorrências obstétricas ocorridas durante a gestação.

\section{MÉTODOS}

O estudo apresentou caráter retrospectivo, descritivo de corte transversal e análise quantitativa. O primeiro contato da população com o serviço de saúde é recomendado que seja pela Estratégia de Saúde da Família ela é responsável pela organização do atendimento, sua ênfase está nas ações de promoção, prevenção, atenção e reabilitação da saúde. É estruturada e centralizada na saúde da família com foco não apenas curativo, mas na prevenção da doença. A constituição da ESF é recomendada que seja no mínimo médico, enfermeiro, auxiliar de enfermagem e agentes comunitários de saúde (SECRETARIA MUNICIPAL DE SAÚDE, 2017). No ano de 2020, o município onde foi realizado o estudo contava com 137 Equipes de Saúde da Família, sendo 127 na área urbana e 10 na área rural (MINISTÉRIO DA SAÚDE, 2020).

As participantes foram identificadas através do auxílio dos enfermeiros e dos agentes comunitários de saúde, bem como através de busca ativa, por meios digitais. Participaram da pesquisa as mulheres maiores de 18 anos, que tiveram gravidez e parto nos últimos seis meses e que fossem cadastradas e acompanhadas pela Estratégia Saúde da Família em de um município do Norte de Minas Gerais, e foram excluídas as mulheres menores de 18 anos.

Foi utilizado como instrumento o Google Formulários com a elaboração de um questionário desenvolvido pelas próprias pesquisadoras a partir das informações disponíveis em artigos científicos que abordaram a temática, que obteve questões sobre informações sociodemográficas, tais como idade, estado civil, renda familiar, escolaridade, raça, ocupação. Sobre as questões obstétricas, foram abordadas questões sobre infecção urinária, diabetes gestacional, anemia, hemorragias, doença hipertensiva da gestação, doenças infecciosas, trabalho de parto prematuro, toxoplasmose e sífilis. Direcionadas às mulheres que tiveram gravidez e parto nos últimos seis meses, onde elas responderam o questionário para a construção da base de dados. Foi realizado um pré-teste com mulheres não incluídas nos critérios de inclusão de pesquisa, com o objetivo de testar eventuais falhas no instrumento.

As participantes foram identificadas com o auxílio dos Agentes Comunitários de Saúde que indicaram as mulheres que se encaixaram nos critérios de inclusão e em seguida foi iniciada a coleta de dados com o envio do formulário eletrônico por rede social e/ou e-mail. Antes de ter acesso ao questionário, as participantes assinavam a opção de concordância com o Termo de Consentimento Livre Esclarecido (TCLE) e em seguida eram direcionadas para as questões específicas da pesquisa.

As respostas das participantes eram direcionadas automaticamente para uma planilha do Excel e após a finalização da coleta, os dados foram exportados para o software estatístico IBM Statistical Package for the Social Sciences (SPSS) for Windows, versão 22.0, que foi utilizado para análise dos dados. Os resultados foram apresentados com seus valores absolutos ( $n$ ) e relativos (\%).

A pesquisa respeitou as normas e diretrizes da Resolução 466/2012 que trata pesquisa com seres humanos e foi submetida ao CEP (Comitê de Ética em Pesquisa) da Soebras, sendo aprovado sob parecer de número 4.284.829.

\section{RESULTADOS}

Participaram da pesquisa 22 mulheres entre 21 e 40 anos, com média de idade de 30 anos. A maioria está na faixa etária de 26 a 35 anos (54,5\%). Quanto à cor e estado civil 72,7\% eram da cor Parda e 63,6\% eram casadas. O grau de escolaridade que predominou foi ensino médio com $54,5 \%$. Obteve 11 participantes que são assalariados/empregadas (63,6\%) e 45,5\% com renda de 1 a 3 salários mínimos. Do total das participantes, $22,7 \%$ tinham uma carga horária de 40 horas ou mais (Tabela 1). 
Tabela 1 - Dados sociodemográficos e econômicos das participantes.

\begin{tabular}{|c|c|c|}
\hline Variáveis & $\mathbf{N}$ & $\%$ \\
\hline \multicolumn{3}{|l|}{ Idade } \\
\hline De 19 a 25 anos & 6 & 27,3 \\
\hline De 26 a 35 anos & 12 & 54,5 \\
\hline 36 anos ou mais & 4 & 18,2 \\
\hline \multicolumn{3}{|l|}{ Cor } \\
\hline Branca & 5 & 22,7 \\
\hline Parda & 16 & 72,7 \\
\hline Preta & 1 & 4,5 \\
\hline \multicolumn{3}{|l|}{ Estado civil } \\
\hline Casada & 14 & 63,6 \\
\hline Solteira & 2 & 9,1 \\
\hline União consensual & 6 & 27,3 \\
\hline \multicolumn{3}{|l|}{ Escolaridade } \\
\hline Fundamental & 1 & 4,5 \\
\hline Médio & 12 & 54,5 \\
\hline Pós graduação & 3 & 13,6 \\
\hline Superior & 6 & 27,3 \\
\hline \multicolumn{3}{|l|}{ Ocupação } \\
\hline Dona de casa & 5 & 22,7 \\
\hline Assalariada/empregada & 14 & 63,6 \\
\hline Trabalha por conta própria & 1 & 4,5 \\
\hline Nenhuma & 2 & 9,1 \\
\hline \multicolumn{3}{|l|}{ Renda } \\
\hline Até 1 salário mínimo & 7 & 31,8 \\
\hline Entre 1 e 3 salários mínimos & 10 & 45,5 \\
\hline \multicolumn{3}{|c|}{ Carga horária de trabalho semanal } \\
\hline Até 40 horas semanais & 9 & 40,9 \\
\hline Mais de 40 horas semanais & 5 & 22,7 \\
\hline
\end{tabular}

Fonte: Sá AP, et al., 2021.

O número prevalente de gestação, parto e aborto entre as mulheres foram de 1 gestação $(36,4 \%) 1$ parto $(50,0 \%)$ e nenhum aborto $(77,3 \%)$. Durante a gravidez a maioria das mulheres realizaram 4 ou mais exames de sangue $(45,5 \%), 8$ ou mais consultas de pré-natal $(68,2 \%)$ e $68,2 \%$ fizeram 4 ou mais exame de ultrassonografia. $77,3 \%$ das participantes fizeram uso de algum medicamento durante a gravidez, dentre eles os mais usados foram metildopa, utrogestan e antibióticos $(9,1 \%)$. O número de internações foi relativamente baixo nessa pesquisa com 18,2\%. Predominou o ganho de 7 a 12 quilos durante a gestação (68,2\%). 47,4\% das mulheres tiveram problemas durante a gravidez. Todas as participantes desta pesquisa fizeram o uso de sulfato ferroso ou outro suplemento que contém ferro e de ácido fólico ou outro suplemento que contém ácido e nenhuma teve gestação gemelar (Tabela 2). 
Tabela 2 - Dados da gestação.

\begin{tabular}{|c|c|c|}
\hline Variáveis & $\mathbf{N}$ & $\%$ \\
\hline \multicolumn{3}{|l|}{ Número de gestações } \\
\hline 1 & 8 & 36,4 \\
\hline 2 & 5 & 22,7 \\
\hline 3 & 7 & 31,8 \\
\hline \multicolumn{3}{|l|}{ Número de Partos } \\
\hline 1 & 11 & 50,0 \\
\hline 2 & 5 & 22,7 \\
\hline 3 & 4 & 18,2 \\
\hline 4 ou mais & 2 & 9,1 \\
\hline \multicolumn{3}{|l|}{ Número de Abortos } \\
\hline 0 & 17 & 77,3 \\
\hline 1 & 4 & 18,2 \\
\hline 2 & 1 & 4,5 \\
\hline \multicolumn{3}{|l|}{ Exame de sangue durante a gravidez } \\
\hline realizou 2 vezes & 6 & 27,3 \\
\hline realizou 3 vezes & 6 & 27,3 \\
\hline realizou 4 vezes ou mais & 10 & 45,5 \\
\hline \multicolumn{3}{|l|}{ Consultas de pré-natal } \\
\hline 1 a 3 consultas & 1 & 4,5 \\
\hline 4 a 5 consultas & 1 & 4,5 \\
\hline 6 a 7 consultas & 5 & 22,7 \\
\hline 8 ou mais consultas & 15 & 68,2 \\
\hline \multicolumn{3}{|l|}{ Ultrassonografia durante a gravidez } \\
\hline Não & 1 & 4,5 \\
\hline realizou 2 vezes & 4 & 18,2 \\
\hline realizou 3 vezes & 2 & 9,1 \\
\hline realizou 4 vezes ou mais & 15 & 68,2 \\
\hline \multicolumn{3}{|c|}{ Uso de algum medicamento na gravidez } \\
\hline Não & 5 & 22,7 \\
\hline Sim & 17 & 77,3 \\
\hline \multicolumn{3}{|c|}{ Se você fez uso de medicamento na gravidez, qual foi? } \\
\hline Metildopa & 2 & 9,1 \\
\hline Utrogestan & 2 & 9,1 \\
\hline Antibiotico & 2 & 9,1 \\
\hline Insulina+sertralina & 1 & 4,5 \\
\hline Metildopa+AAS & 1 & 4,5 \\
\hline Metformina+enoxaparina+Utrogestan & 1 & 4,5 \\
\hline \multicolumn{3}{|l|}{ Internação na gestação } \\
\hline Não & 18 & 81,8 \\
\hline Sim, apenas uma vez & 4 & 18,2 \\
\hline \multicolumn{3}{|l|}{ Ganho de peso } \\
\hline Menos de 6 quilos & 1 & 4,5 \\
\hline Entre 7 e 12 quilos & 15 & 68,2 \\
\hline Entre 13 e 15 quilos & 4 & 18,2 \\
\hline 16 kilos ou mais & 2 & 9,1 \\
\hline
\end{tabular}

Fonte: Sá AP, et al., 2021.

Como pode ser observado na Tabela 3 , em relação às principais intercorrências foi verificado que $27,3 \%$ das participantes tiveram infecção urinária durante a gravidez e 27,3\% tiveram diabetes gestacional. A anemia esteve presente em $13,6 \%$ das participantes, enquanto as hemorragias $4,5 \%$. Sobre a doença hipertensiva da gestação $22,7 \%$ tiveram, e a toxoplasmose teve apenas $4,5 \%$. Em relação a doenças infecciosas, três participantes afirmaram que tiveram candidíase durante a gravidez (13,6\%). Já o trabalho de parto prematuro e os distúrbios do líquido amniótico foram uma realidade de $4,5 \%$ das gestações. Nenhuma das participantes nunca teve Sífilis e placenta prévia. Dentre outras intercorrências prevaleceu o colo do útero curto com $(4,5 \%)$. 
Tabela 3 - Intercorrências da gestação.

\begin{tabular}{|c|c|c|}
\hline Variáveis & $\mathbf{N}$ & $\%$ \\
\hline \multicolumn{3}{|l|}{ Infecção urinária } \\
\hline Nunca tiveram & 10 & 45,5 \\
\hline Tiveram antes da gravidez & 6 & 27,3 \\
\hline Tiveram durante a gravidez & 6 & 27,3 \\
\hline \multicolumn{3}{|l|}{ Diabetes gestacional } \\
\hline Nunca tiveram & 15 & 68,2 \\
\hline Tiveram antes da gravidez & 1 & 4,5 \\
\hline Tiveram durante a gravidez & 6 & 27,3 \\
\hline \multicolumn{3}{|l|}{ Anemia } \\
\hline Nunca tiveram & 17 & 77,3 \\
\hline Tiveram antes da gravidez & 2 & 9,1 \\
\hline Tiveram anemia durante a gravidez & 3 & 13,6 \\
\hline \multicolumn{3}{|l|}{ Hemorragias } \\
\hline Nunca tiveram & 20 & 90,9 \\
\hline Tiveram antes da gravidez & 1 & 4,5 \\
\hline Tiveram durante a gravidez & 1 & 4,5 \\
\hline \multicolumn{3}{|l|}{ Doença hipertensiva } \\
\hline Nunca tiveram & 15 & 68,2 \\
\hline Tiveram antes da gravidez & 1 & 4,5 \\
\hline Tiveram durante a gravidez e continuou após o parto & 1 & 4,5 \\
\hline Tiveram durante a gravidez & 5 & 22,7 \\
\hline \multicolumn{3}{|l|}{ Toxoplasmose } \\
\hline Nunca tiveram & 20 & 90,9 \\
\hline Tiveram antes da gravidez & 1 & 4,5 \\
\hline Tiveram durante a gravidez & 1 & 4,5 \\
\hline \multicolumn{3}{|l|}{ Doenças infecciosas } \\
\hline Nunca tiveram & 19 & 84,4 \\
\hline Tiveram antes da gravidez & 3 & 13,6 \\
\hline \multicolumn{3}{|l|}{ Trabalho de parto prematuro } \\
\hline Nunca tiveram & 18 & 81,8 \\
\hline Tiveram em gestações anteriores & 1 & 4,5 \\
\hline Tiveram na última gestação & 3 & 13,6 \\
\hline \multicolumn{3}{|l|}{ Distúrbios do líquido amniótico } \\
\hline Nunca tiveram & 19 & 86,4 \\
\hline Tiveram em gestações anteriores & 1 & 4,5 \\
\hline Tiveram na última gestação & 2 & 9,1 \\
\hline \multicolumn{3}{|l|}{ Outras. Quais? (descreva) } \\
\hline Colo do útero curto & 1 & 4,5 \\
\hline
\end{tabular}

Fonte: Sá AP, et al., 2021. 


\section{DISCUSSÃO}

Os resultados desta pesquisa demonstraram que as principais intercorrências na gestação foram a hipertensão, diabetes e infecção urinária. Na pesquisa realizada por Calegari RS, et al. (2016) com puérperas em um hospital universitário de Porto Alegre, observou-se que $67,9 \%$ das mulheres tiveram alguma intercorrência e a mais frequente foi a infecção do trato urinário.

Embora a melhoria na qualidade da assistência ao pré-natal, parto e puerpério impactem diretamente nos índices de mortalidade materna, é pertinente que tenha outras estratégias a serem executadas para garantir uma certificar reduzir o número de óbitos maternos. Investimentos de cunho social, direcionado a políticas públicas para educação básica, redução da pobreza e das desigualdades sociais, se fazem necessários por serem aptos a interferir nas circunstâncias de vida e bem estar das mulheres (SANTOS LO, et al., 2021).

Através da pesquisa realizada por Pavelqueires S e Santos ITRS (2020), obteve dados significativos de tratamentos realizados pelas gestantes e puérperas atendidas em urgência e emergência obstétrica no interior paulista de janeiro 2014 a abril 2018, na qual destacou-se a quantidade de tratamentos realizados por intercorrências na gravidez (92,8\%).

De acordo com Faleiros GQA, et al. (2021). As dosagens das glicemias plasmáticas permitiram perceber que as gestantes diabéticas gestacionais apresentaram glicemias mais elevadas no primeiro, segundo e terceiro trimestres de gestação. De modo respectivo, o que é esperado tendo em consideração a patologia estudada. Conduto as médias de glicemias, constatou que a queda ao longo dos trimestres gestacionais está relacionada ao pré-natal. Um pré-natal eficiente, equipe treinada e exames disponíveis são suficientes para fazer a diferença no prognóstico desses pacientes.

No entanto, com base nos estudos do autor Cantalixto VF e Farias FN (2021) foram apresentadas e avaliadas as 85 mortes de mulheres que residiam em Juiz de Fora. O estudo abrangeu a faixa etária entre 20 e 36 anos. Os resultados das pacientes que constataram o pré-natal foram de $(74,1 \%)$, e as pacientes com menos de seis avaliações foram (34,0\%). A cesariana foi realizada em $38,8 \%$ dos partos, e em tratamento obstétrico $(32,9 \%)$ obtiveram o procedimento apropriado. A primeira morte materna constatada foi a de choque hipovolêmico 12 (14,10\%), em seguida foi averiguado morte por hipotonia uterina $(7,0 \%)$.

Segundo Cirqueira RS, et al. (2018), houve concordância com essa pesquisa apenas o fato da infecção urinária ser a complicação predominante $(20 \%)$, havendo discrepância a anemia $(18,4 \%)$ em ser a segunda mais frequente. Enquanto os casos de hipertensão (4,3\%) e diabetes mellitus $(1,1 \%)$ apresentaram menor prevalência discordando dessa pesquisa.

Concordando com essa pesquisa dentre as alterações mais frequentes, estão infecções do trato urinário (ITU) (38,2\%), a doença hipertensiva da gestação (DHEG) (19,5\%), e diabetes mellitus gestacional, além delas também está presente à anemia com $26,0 \%$ e o trabalho de parto prematuro (TPP) com 22,6\% (VARELA PLR, et al., 2017).

O diabetes mellitus gestacional ocupa a categoria de um dos maiores problemas de saúde pública, com até $37,7 \%$ dos casos no mundo, sendo $18 \%$ no Brasil (OPAS, 2016). Essa doença requer atenção profissional devido a alterações no desenvolvimento intra-uterino, podendo levar a anomalias congênitas e consequentemente ao risco de aborto. Durante o desenvolvimento fetal pode ocorrer macrossomia, icterícia e evoluir à morte (OLIVEIRA ACM, et al., 2016).

De acordo com Magalhães EIS, et al. (2018), os resultados do presente estudo mostram que a anemia nas gestantes assistidas em unidades de saúde da zona urbana do município de Vitória da Conquista é um problema de saúde pública. As integrações de hemoglobina nessa população são determinadas por causas obstétricas e relacionadas à assistência pré-natal. As circunstâncias, salientam que é de suma importância da atenção básica no cuidado à saúde da gestante, o que acrescentar desde o acesso à educação em saúde bem como a disponibilização de exames complementares e suplementos de ferro e medicamentos para 0 cuidado a agravos de saúde, como a anemia. No entanto, os estudos sobre o tema são de grande relevância, uma vez que seus resultados podem se direcionar à implementação de ações de saúde pública que visa à manutenção e ao comando da concentração de hemoglobina em níveis apropriados e também mediante a prevenção da anemia na gestação. 
Conforme Luz AG, et al. (2014), seguindo todos os cuidados prescritos no pré natal as ITUs podem ser prevenível, e sua importância se dá por ser um dos assuntos e relatos mais comuns dentre as gestantes, com periodicidade de 31,5\% ultrapassando sua média (VARELA PLR, et al., 2017; MORDI RM, et al, 2015). A ITU na maioria das vezes pode ser assintomática e por isso muitas mulheres não procuram tratamento, se não tratada tem um potencial risco para pielonefrite e cistite, trazendo sérios prejuízos para o feto como seu nascimento antes de completar as 37 semanas, e seu período perinatal pequeno para idade gestacional (PIG) (MINISTÉRIO DE SAÚDE, 2012).

De acordo com Nascimento DS, et al. (2021) a consulta de pré-natal realizada na Atenção Primária à Saúde é indispensável para a gestação sem complicações e deve ser iniciada sucessivamente após um resultado positivo de gravidez. Referente ao PN, quanto maior for, menor é o risco de complicações durante a gestação e puerpério. No estudo feito, foi comprovado a sua relevância, pois $86 \%$ das gestantes que aderiram ao pré-natal, não complicaram, comparado aos $13 \%$ das gestantes que têm complicações na gestação, as causa; sangramentos, convulsão, infecção urinária, diabetes e hipertensão arterial.

O Brasil está como décimo país do mundo, em relação a dados da OMS com número absoluto de nascimentos pré-termo. A estimativa dos autores Souza MF, et al. (2019) para a prevalência de prematuridade no Brasil é de 9,2\%. Por meio dos dados coletados do Sistema Nacional de Nascidos Vivos (SINASC) entre 2000 e 2010, indicam um discreto aumento da prevalência de nascidos pré-termo durante esse período, de $6,8 \%$ para $7,2 \%$. Conforme alguns estudos nacionais, a prevalência de prematuridade ultrapassa informações estimadas pelo SINASC, sendo consideravelmente maior nas últimas décadas.

Neste estudo, foram poucas as gestantes que apresentaram candidíase, contudo neste público há uma maior susceptibilidade a candidíase vulvovaginal, devido aos níveis elevados de estrógeno e progesterona presentes durante a gravidez. A Candida albicans, gênero com maior prevalência de infecção, apresenta uma proteína de ligação de estrógeno o que causa uma maior interação entre ambos (NUNES AMA, 2021).

Waikhom SD, et al. (2020) afirma que a candidíase pode apresentar um risco maior às gestantes pela falta de cuidado e interesse em procurar auxílio médico. Diante do risco apresentado, a candidíase está relacionada a problemas fetais, apresentando, assim, um grande risco à gestação. Outro ponto questionado é que falta um melhor acompanhamento no sistema público de saúde no que tange ao cuidado pré-natal e o debate de infecções do trato vaginal.

As participantes da pesquisa realizada por Magalhães EIS, et al. (2018) atenderam nas unidades de saúde urbanas de Vitória da Conquista, na Bahia, dentre elas houve uma prevalência verificada no estudo de 18,9\%, afirmando também que a anemia é um problema de saúde pública. A anemia na gestação pode implicar prejuízos como baixo peso, mortalidade perinatal, aumento de risco de aborto espontâneo, trabalho de parto prematuro. Está associada a baixa escolaridade, renda baixa, privação de ferro, alimentação inadequada e mulheres multíparas (CAMPIGOTTO AC, et al., 2015; OMS, 2013).

A pesquisa apresentou limitações como o impedimento do contato direto com as participantes, devido a pandemia. A dificuldade apresentada foi na identificação das parturientes, na qual tivemos restrições com alguns profissionais de saúde pelo fato de ficarem inseguros ou não conseguirem o telefone das mesmas. Outro fato de dificuldade foi devido à padronização utilizada por outros artigos, e também na comparação de resultados com outras pesquisas devido às diferentes formas de abordagem das variáveis.

\section{CONCLUSÃO}

Foi considerado um número significativo de mulheres que tiveram algum tipo de intercorrência durante a gravidez, sendo fator de risco para predispor a outras complicações. Nessa pesquisa constatou-se que as intercorrências que mais acometeram as participantes foram infecção urinária, diabetes mellitus gestacional e distúrbios hipertensivos. Todas elas podem ser evitadas ou controladas com um bom monitoramento durante as consultas de pré-natal. Através da análise de dados foi possível perceber que as intercorrências encontradas na amostra pesquisada mantêm o mesmo padrão de outros estudos realizados no Brasil. A partir desses resultados sugere-se novas pesquisas que abordem o tema com avaliação dos fatores associados às intercorrências prevalentes na gestação. 


\section{REFERÊNCIAS}

1. BUCHANAN TA, et al. Diabetes Mellitus Gestacional: Riscos e Manejo durante e após a Gravidez. Nat Rev Endocrinol, 2012; (8): 639-649.

2. CALEGARI RS et al. Intercorrências Clínicas e Obstétricas vivenciadas por mulheres no Pré-Natal. Congitare Enferm, 2016; 21(2): 1-8.

3. CAMPIGOTTO AC, et al. Fatores Relacionados à Deficiência de Ferro Anemia na gravidez: uma Revisão Integrativa. Revistas do iMed, 2015; 8(159).

4. CANTALIXTO VF, FARIAS FN. Conceitos e características da morbidade materna e near miss: revisão bibliográfica. Revista Eletrônica Acervo Saúde, 2021; 13 (1): e5752-e5752.

5. CIRQUEIRA GC, et al. Intercorrências no primeiro trimestre: assistência ao pré-natal nas unidades de saúde da família do recôncavo baiano. Revista Brasileira de Saúde Funcional (REBRASF). 2018; 6(1).

6. FALEIROS GQA, et al. Diabetes Mellitus Gestacional: o controle glicêmico como elemento de controle de peso fetal. Revista Eletrônica Acervo Saúde, 2021; 13(5): 2178-2091.

7. LUZ AG, et al. Impacto de um estudo nacional para a vigilância do near miss materno na qualidade da assistência prestada pelos centros participantes: uma abordagem quantitativa e qualitativa. BMC Gravidez Parto, 2014; 14(122).

8. MAGALHÃES EIS, et al. Prevalencia de anemia e determinantes da concentração de hemoglobina em gestantes. Cad Saúde Colet, 2018; 26(4): 384-390.

9. MINISTÉRIO DA SAÚDE. Atenção ao pré-natal de baixo risco. Brasília: Brasil, 2012a. Disponível em: https://bvsms.saude.gov.br/bvs/publicacoes/cadernos_atencao_basica_32_prenatal.pdf Acesso em: 22 mar 2020 às 14:00

10. MINISTÉRIO DA SAÚDE. Caderneta da Gestante. 4 edição, Brasília-DF: Brasil, 2018a. Disponível em: https://portalarquivos2.saude.gov.br/images/pdf/2018/agosto/31/Caderneta-da-Gestante-2018.pdf Acesso em: 19 set. 2021 às 16:19.

11. MINISTÉRIO DA SAÚDE. Fundação Oswaldo Cruz (Fiocruz). Hemorragia pós-parto. Portal de Boas Práticas em Saúde da Mulher, da Criança e do Adolescente. Rio de Janeiro: Brasil, 2018b. Disponível em: https://www.arca.fiocruz.br/bitstream/icict/29895/2/HEMORRAGIA\%20P\%c3\%93S-PARTO.pdf Acesso em: 22 mar 2020 às $14: 30$.

12. MINISTÉRIO DE SAÚDE. Gestação de alto risco: manual técnico. Brasília: Brasil, 2012b. Disponivel em: https://bvsms.saude.gov.br/bvs/publicacoes/manual_tecnico_gestacao_alto_risco.pdf acesso em:10 mar 2020 ás 18:00.

13. MINISTÉRIO DA SAÚDE. Protocolos da Atenção Básica: Saúde das Mulheres. Brasília: Ministério da Saúde, 2016. Disponível em: https://bvsms.saude.gov.br/bvs/publicacoes/protocolos_atencao_basica_saude_mulheres.pdf Acesso em: 23 mar 2020 ás 15:00.

14. MINISTÉRIO DA SAÚUDE. Secretaria de Atenão Primária à Saúde. E-GESTOR Atenção Básica à Saúde. Brasilia: Brasil, 2020. Disponível em: BRASIL. Secretaria de Atenção Primária à Saúde. Brasília: Ministério da saúde. Disponível em: https://egestorab.saude.gov.br/paginas/acessoPublico/relatorios/relHistoricoCoberturaAB.xhtml. Acesso em: 09 mai. às 15:33.

15. MINISTÉRIO DA SAÚDE. Secretaria de Políticos de Saúde. Área Técnica de Saúde da Mulher. Parto, aborto e puerpério: assistência humanizada à mulher/ Ministério da Saúde, Secretaria de Políticas de Saúde, Área Técnica da Mulher. - Brasília: Brasil, 2001. Disponível em: https://bvsms.saude.gov.br/bvs/publicacoes/cd04_13.pdf Acesso em: 27 set. às $22: 25$.

16. MORDI RM, et al. Prevalence of urinary tract infections (UTI) Among pregnant women in univeersity of benin teaching hospital (UBTH) Benin City, Nigeria. Journal of Asian Scientific Research, 2015; 5(4): 198-204.

17. MOURA BLA, et al. Internações por complicações obstétricas na gestação e desfechos maternos e perinatais, em uma coorte de gestantes no Sistema Único de Saúde no Município de São Paulo, Brasil. Cad. Saúde Pública 2018; 34(1): e00188016.

18. NASCIMENTO DS, et al. Assistência de enfermagem ao pré-natal na atenção básica: uma revisão integrativa. Revista Artigos.Com, 2021; 27: e7219-e7219.

19. NUNES AMA, et al. Avaliação da infecção do trato urinário em gestantes e acompanhamento farmacoterapêutico. Journal of Biology \& Pharmacy and Agricultural Management, 2021; 17(3).

20. OLIVEIRA ACM, et al. Fatores maternos e resultados perinatais adversos em mulheres com pré-eclâmpsia em Maceió, Alagoas. Arq Bras Cardiol, 2016; 106(2): 113-120.

21. ORGANIZAÇÃO MUNDIAL DA SAÚDE (OMS). Diretriz: Suplementação diária de ferro e ácido fólico em gestantes. Genebra: Organização Mundial da Saúde, $2013 . \quad$ Disponível em:http://189.28.128.100/dab/docs/portaldab/documentos/guia_gestantes.pdf. Acesso em 29 de abril 2020 ás 15:30.

22. ORGANIZAÇÃO PAN-AMERICANA DA SAÚDE (OPAS), ORGANIZAÇÃOO MUNDIAL DA SAÚDE (OMS). Folha informativa: Mortalidade materna. $2018 . \quad$ Disponível https://www.paho.org/bra/index.php?option=com_content\&amp;view=article\&amp;id=5741 :folha-informativamortalidade-materna\&amp;Itemid=820. Acesso em: 19 mar. 2020 às 18:18 
23. ORGANIZAÇÃO PAN-AMERICANA DA SAÚDE (OPAS). Rastreamento e diagnóstico de diabetes mellitus gestacional no Brasil. Brasília, DF: OPAS, 2016. Disponível em : https://iris.paho.org/handle/10665.2/34278?show=full. Acesso em abril 2020 ás 19:00.

24. PAVELQUEIRES S, SANTOS ITR. Perfil de gestantes atendidas em um hospital público no interior paulista. Revista Eletrônica Acervo Enfermagem, 2020; (4): 2674-7189.

25. SANTOS LO, et al. Estudo da mortalidade materna no Nordeste Brasileiro, de 2009 a 2018. Revista Eletrônica Acervo Saúde, 2021; 13(2): e5858-e5858.

26. SECRETARIA DE SAÚDE DO PARANÁ. Linha Guia. Rede Mãe Paranaense, Paraná, 2018; 7. Disponível em https://www.saude.pr.gov.br/sites/default/arquivos_restritos/files/documento/202009/LinhaGuiaMaeParanaense_2018.pdf Acesso 19 de abril de 2020 ás 19:00.

27. SECRETARIA MUNICIPAL DE SAÚDE. Protocolo assistencial de enfermagem em saúde da mulher: assistência à gestante do município de Montes Claros, 2017; 2. Disponível em:

http://www.montesclaros.mg.gov.br/diariooficial/2018/jan-18/Anexo\%20Portaria\%20SMS\%2012-

2018/fwprotocolosenfermagem4/PROTOCOLO\%20ENF\%20PRE\%20NATAL.pdf. Acesso em: 27 de abril ás 17:00.

28. SHELDON WR, et al. Manejo da hemorragia pós-parto, riscos e resultados maternos: resultados da Pesquisa Multicountry da Organização Mundial de Saúde sobre Saúde Materna e do Recém-Nascido. BJOG: um International Journal of Obstetrics \& Gynecology, 2014; 121(Suppl.1): 5-13.

29. SILVA EP, et al. Impacto de estratégias educacionais no pré-natal de baixo risco: revisão sistemática de ensaios clínicos randomizados. Ciência \& Saúde Coletiva, 2016; 21: 2935-2948.

30. SOUZA MF, et al. Sistematização da Assistência de Enfermagem ao trabalho de parto prematuro: Um estudo de caso. Braz. J. of Develop, 2019; 5(12): 32974-32983.

31. VARELA PLR, et al. Intercorrências na gravidez em puérperas brasileiras atendidas nos sistemas público e privado de saúde. Latino-Am Enfermagem, 2017; 25.

32. WAIKHOM SD, et al. Prevalência de candidíase vulvovaginal em mulheres grávidas no município de Ho, Gana: identificação de espécies e susceptibilidade antifúngica de isolados de Candida. BMC Pregnancy Childbirth 2020; 20(266). 